\title{
On Generalized Semiderivations of Prime Near Rings
}

\author{
Abdelkarim Boua, ${ }^{1}$ A. Raji, ${ }^{2}$ Asma Ali, ${ }^{3}$ and Farhat Ali $^{3}$ \\ ${ }^{1}$ Department of Mathematics, Faculty of Sciences of Agadir, Ibn Zohr University, P.O. Box 8106, Agadir, Morocco \\ ${ }^{2}$ Département de Mathématiques, Faculté des Sciences et Techniques, Université Moulay Ismaïl, \\ Groupe d'Algèbre et Applications, BP 509, Boutalamine, Errachidia, Morocco \\ ${ }^{3}$ Department of Mathematics, Aligarh Muslim University, Aligarh 202002, India
}

Correspondence should be addressed to Asma Ali; asma_ali2@rediffmail.com

Received 6 August 2015; Accepted 1 October 2015

Academic Editor: Kaiming Zhao

Copyright (c) 2015 Abdelkarim Boua et al. This is an open access article distributed under the Creative Commons Attribution License, which permits unrestricted use, distribution, and reproduction in any medium, provided the original work is properly cited.

\begin{abstract}
Let $N$ be a near ring. An additive mapping $F: N \rightarrow N$ is said to be a generalized semiderivation on $N$ if there exists a semiderivation $d: N \rightarrow N$ associated with a function $g: N \rightarrow N$ such that $F(x y)=F(x) y+g(x) d(y)=d(x) g(y)+x F(y)$ and $F(g(x))=g(F(x))$ for all $x, y \in N$. In this paper we prove that prime near rings satisfying identities involving semiderivations are commutative rings, thereby extending some known results on derivations, semiderivations, and generalized derivations. We also prove that there exist no nontrivial generalized semiderivations which act as a homomorphism or as an antihomomorphism on a 3-prime near ring $N$.
\end{abstract}

\section{Introduction}

Throughout the paper, $N$ denotes a zero-symmetric left near ring with multiplicative centre $Z$; and for any pair of elements $x, y \in N,[x, y]$ denotes the commutator $x y-y x$ while the symbol $(x, y)$ denotes the additive commutator $x+y-$ $x-y$. An element $x$ of $N$ is said to be distributive if $(y+$ $z) x=y x+z x$, for all $y, z \in N$. A near ring $N$ is called zero-symmetric if $0 x=0$, for all $x \in N$ (recall that left distributivity yields that $x 0=0$ ). The near ring $N$ is said to be 3-prime if $x N y=\{0\}$ for $x, y \in N$ implies that $x=0$ or $y=0$. A near ring $N$ is called 2-torsion free if $(N,+)$ has no element of order 2. An additive mapping $f: N \rightarrow N$ is said to be a right (resp., left) generalized derivation with associated derivation $D$ if $f(x y)=f(x) y+x D(y)$ (resp., $f(x y)=D(x) y+x f(y))$, for all $x, y \in N$, and $f$ is said to be a generalized derivation with associated derivation $D$ on $N$ if it is both a right generalized derivation and a left generalized derivation on $N$ with associated derivation $D$. Motivated by a definition given by Bergen [1] for rings, we define an additive mapping $d: N \rightarrow N$ to be a semiderivation on a near ring $N$ if there exists a function $g: N \rightarrow N$ such that (i) $d(x y)=d(x) g(y)+x d(y)=d(x) y+g(x) d(y)$ and (ii) $d(g(x))=g(d(x))$, for all $x, y \in N$. In case $g$ is the identity map on $N, d$ is of course just a derivation on $N$, so the notion of semiderivation generalizes that of derivation. But the generalization is not trivial; for example, take $N=$ $N_{1} \oplus N_{2}$, where $N_{1}$ is a zero-symmetric near ring and $N_{2}$ is a ring. Then the map $d: N \rightarrow N$ defined by $d((x, y))=(0, y)$ is a semiderivation associated with function $g: N \rightarrow N$ such that $g(x, y)=(x, 0)$. However $d$ is not a derivation on $N$. An additive mapping $F: N \rightarrow N$ is said to be a generalized semiderivation of $N$ if there exists a semiderivation $d: N \rightarrow$ $N$ associated with a map $g: N \rightarrow N$ such that (i) $F(x y)=$ $F(x) y+g(x) d(y)=d(x) g(y)+x F(y)$ and (ii) $F(g(x))=$ $g(F(x))$ for all $x, y \in N$. All semiderivations are generalized semiderivations. Moreover, if $g$ is the identity map on $N$, then all generalized semiderivations are merely generalized derivations; again the notion of generalized semiderivation generalizes that of generalized derivation. Moreover, the generalization is not trivial as the following example shows.

Example 1. Let $S$ be a 2-torsion free left near ring and let

$$
N=\left\{\left(\begin{array}{lll}
0 & x & y \\
0 & 0 & 0 \\
0 & 0 & z
\end{array}\right) \mid x, y, z \in S\right\} .
$$


Define maps $F, d, g: N \rightarrow N$ by

$$
\begin{aligned}
& F\left(\begin{array}{lll}
0 & x & y \\
0 & 0 & 0 \\
0 & 0 & z
\end{array}\right)=\left(\begin{array}{ccc}
0 & x y & 0 \\
0 & 0 & 0 \\
0 & 0 & 0
\end{array}\right) ; \\
& d\left(\begin{array}{lll}
0 & x & y \\
0 & 0 & 0 \\
0 & 0 & z
\end{array}\right)=\left(\begin{array}{lll}
0 & 0 & y \\
0 & 0 & 0 \\
0 & 0 & z
\end{array}\right), \\
& g\left(\begin{array}{lll}
0 & x & y \\
0 & 0 & 0 \\
0 & 0 & z
\end{array}\right)=\left(\begin{array}{lll}
0 & x & 0 \\
0 & 0 & 0 \\
0 & 0 & 0
\end{array}\right) .
\end{aligned}
$$

It can be verified that $N$ is a left near ring and $F$ is a generalized semiderivation with associated semiderivation $d$ and a map $g$ associated with $d$. However $F$ is not a generalized derivation on $N$.

\section{Preliminary Results}

We begin with the following Lemmas which are extensively used to prove our main theorems. Unless it is stated otherwise, it will be assumed that $N$ is a zero-symmetric 3-prime near ring.

Lemma 2 (see [2, Lemma 1.2]). Let $N$ be a 3-prime near ring.

(i) If $z \in Z \backslash\{0\}$ and $x z \in Z$, then $x \in Z$.

(ii) If $x \in Z \backslash\{0\}$ then $x$ is not a zero divisor.

Lemma 3 (see [2, Lemma 1.5]). If $N$ is a 3-prime near ring and $Z$ contains a nonzero left semigroup ideal, then $N$ is a commutative ring.

Lemma 4 (see [3, Theorem 2.1]). Let $N$ be a 2-torsion free 3-prime near ring with a nonzero semiderivation $d$ associated with a map $g$. If $d(N) \subseteq Z$, then $N$ is a commutative ring.

Lemma 5. Let $N$ be a 3-prime near ring admitting a generalized semiderivation $F$ associated with a semiderivation $d$. If $g$ is the map associated with $d$ such that $g(x y)=g(x) g(y)$ for all $x, y \in N$, then $N$ satisfies the following partial distributive laws:

(i) $(F(x) y+g(x) d(y)) z=F(x) y z+g(x) d(y) z$ for all $x, y, z \in N$.

(ii) $(d(x) y+g(x) d(y)) z=d(x) y z+g(x) d(y) z$ for all $x, y, z \in N$.

Proof. (i) Let $x, y, z \in N$, and by defining $F$ we have

$$
\begin{aligned}
F(x y z) & =F(x y) z+g(x y) d(z) \\
& =(F(x) y+g(x) d(y)) z+g(x) g(y) d(z) .
\end{aligned}
$$

On the other hand,

$$
\begin{aligned}
F(x y z) & =F(x) y z+g(x) d(y z) \\
& =F(x) y z+g(x)(d(y) z+g(y) d(z)) \\
& =F(x) y z+g(x) d(y) z+g(x) g(y) d(z) .
\end{aligned}
$$

Combining both expressions of $F(x y z)$, we obtain

$$
\begin{array}{r}
(F(x) y+g(x) d(y)) z=F(x) y z+g(x) d(y) z, \\
\forall x, y, z \in N .
\end{array}
$$

(ii) With a simple calculation of $d((x y) z)=d(x(y z))$, we obtain the required result.

Lemma 6. Let $N$ be a 2-torsion free zero-symmetric 3-prime near ring. If $d$ is a nonzero semiderivation of $N$ associated with a map $g$ which is onto, then $d^{2} \neq 0$.

Proof. Suppose $d^{2}(N)=0$. Then for $x, y \in N$, we may write

$$
\begin{aligned}
0= & d^{2}(x y) \\
= & d(d(x y)) \\
= & d(d(x) y+g(x) d(y)), \quad \forall x, y \in N \\
= & d^{2}(x) y+d(x) d(y)+d(g(x)) d(y) \\
& +g(x) d^{2}(y) \\
= & d(x) d(y)+d(g(x)) d(y) .
\end{aligned}
$$

Note that $g(d(x))=d(g(x))$ and $g$ is onto; we get

$$
2 d(x) d(y)=0, \quad \forall x, y \in N .
$$

Since $N$ is 2 -torsion free, we get

$$
d(x) d(y)=0, \quad \forall x, y \in N
$$

Replacing $y$ by $r y$ in the above relation, we get

$$
\begin{aligned}
d(x) d(r y)=0, & \forall x, y, r \in N . \\
d(x)(d(r) y+g(r) d(y))=0, & \forall x, y, r \in N . \\
d(x) d(r) y+d(x) g(r) d(y)=0, & \forall x, y, r \in N .
\end{aligned}
$$

This implies that

$$
\begin{aligned}
d(x) g(r) d(y) & =0, \quad \forall x, y, r \in N . \\
d(x) r d(y) & =0, \quad \forall x, y, r \in N . \\
d(N) N d(N) & =\{0\} .
\end{aligned}
$$

Thus we obtain that $d=0$, a contradiction.

The following lemma extends results of Herstein [4, Theorem 2] and Bell and Mason [2, Theorem 3]. 
Lemma 7. Let $N$ be a 2-torsion free 3-prime near ring admitting a nonzero semiderivation $d$ and a map $g$ associated with $d$ such that $g$ is onto and $g(x y)=g(x) g(y)$ for all $x, y \in N$. If $[d(x), d(y)]=0$ for all $x, y \in N$, then $N$ is a commutative ring.

Proof. Suppose that

$$
d(x) d(y)=d(y) d(x), \quad \forall x, y \in N .
$$

Replacing $y$ by $y z$ in (11) and using Lemma 5(ii), we obtain

$$
\begin{aligned}
& d(x) d(y) z+d(x) g(y) d(z) \\
& \quad=d(y) z d(x)+g(y) d(z) d(x), \quad \forall x, y, z \in N .
\end{aligned}
$$

Substituting $d(y)$ for $y$ in (12) and using (11), we find that

$$
d(x) d^{2}(y) z=d^{2}(y) z d(x), \quad \forall x, y, z \in N .
$$

Taking $z t$ instead of $z$ in (13) after using (13), we arrive at

$$
d^{2}(y) z[d(x), t]=0, \quad \forall x, y, t, z \in N
$$

which can be rewritten as

$$
d^{2}(y) N[d(x), t]=\{0\}, \quad \forall x, y, t \in N .
$$

In the light of the 3-primeness of $N$, (15) implies that

$$
d^{2}=0
$$

or $d(N) \subseteq Z$.

But $d^{2}=0$ contradicts Lemma 6 , so $d(N)$ is contained in $Z$ and $N$ is a commutative ring by Lemma 4 .

\section{The Condition $[F(N), F(N)]=\{0\}$}

The theorems that we prove in this section are motivated by the results proved in [2, Theorem 2], [5, Theorem 2.1], [6, Theorem 2.1 and 4.1], and [3, Theorem 2.1].

Theorem 8. Let $N$ be a 2-torsion free 3-prime near ring with a generalized semiderivation $F$ associated with a nonzero semiderivation $d$ and onto map $g$ associated with $d$ such that $g(x y)=g(x) g(y)$ for all $x, y \in N$. If $F(N) \subseteq Z$, then $N$ is a commutative ring.

Proof. Assume that

$$
F(x) \in Z, \quad \forall x \in N
$$

Taking into account Lemma 5(i), we have for all $x, n \in N$, $z \in Z$,

$$
\begin{aligned}
F(x z) n & =F(x) z n+g(x) d(z) n, \\
n F(x z) & =n F(x) z+n g(x) d(z) \\
& =F(x) z n+n g(x) d(z) .
\end{aligned}
$$

Since $F(x z) n=n F(x z)$, we have $g(x) d(z) n=n g(x) d(z)$ for all $x, n \in N, z \in Z \backslash\{0\}$. Thus $g(x) d(z) \in Z$ for all $x \in N$. Let $d(Z) \neq\{0\}$. Choosing $z$ such that $d(z) \neq 0$ and noting that $d(z) \in Z$, we have $g(x) \in Z$. Since $g$ is onto, we have $N \subseteq Z$. Hence $N$ is a commutative ring by Lemma 3. On the other hand if $d(z)=0$, then for all $x, y \in N$

$$
\begin{aligned}
& 0=d(F(x y)), \\
& 0=d(F(x) y+g(x) d(y)), \\
& 0=F(x) d(y)+g(x) d^{2}(y)+d(g(x)) g(d(y)), \\
& \forall x, y \in Z .
\end{aligned}
$$

Hence $F(x d(y))=-d(g(x)) g(d(y)) \in Z$ for all $x, y \in N$. Since $g$ is onto, we have $d(x) d(y) \in Z$ for all $x, y \in N$. This implies that

$$
d(x)(d(x) d(y)-d(y) d(x))=0, \quad \forall x, y \in N .
$$

Left multiplying by $d(y)$, we arrive at

$$
\begin{aligned}
d(y) d(x) N(d(x) d(y)-d(y) d(x))= & \{0\}, \\
& \forall x, y \in N .
\end{aligned}
$$

Since $N$ is a 3 -prime near ring, we get

$$
[d(x), d(y)]=0, \quad \forall x, y \in N .
$$

We conclude that $N$ is a commutative ring by Lemma 7 .

Corollary 9 (see [6, Theorem 3.2]). Let $N$ be a 2-torsion free 3-prime near ring. If $N$ admits a nonzero generalized derivation $F$ such that $F(N) \subseteq Z$, then $N$ is a commutative ring.

Theorem 10. Let $N$ be a 3-prime near ring admitting a generalized semiderivation $F$ associated with a nonzero semiderivation $d$ and onto map $g$ associated with $d$ such that $g(x y)=$ $g(x) g(y)$ for all $x, y \in N$. If $[F(N), F(N)]=0$, then $(N,+)$ is abelian.

Proof. Assume that

$$
F(x) F(y)=F(y) F(x), \quad \forall x, y \in N
$$

Then

$$
F(x+x) F(y+z)=F(y+z) F(x+x),
$$

$$
\forall x, y, z \in N \text {. }
$$

By (23), the last equation yields that

$$
\begin{aligned}
& F(y) F(x+x)+F(z) F(x+x) \\
& \quad=F(x) F(y+z)+F(x) F(y+z),
\end{aligned}
$$


Hence,

$$
\begin{aligned}
F(y) & F(x)+F(y) F(x)+F(z) F(x)+F(z) F(x) \\
= & F(x) F(y)+F(x) F(z)+F(x) F(y) \\
& +F(x) F(z),
\end{aligned}
$$

that is $F(y) F(x)+F(z) F(x)$

$$
=F(x) F(z)+F(x) F(y), \quad \forall x, y, z \in N,
$$

which implies that

$$
F(y+z-y-z) F(x)=0, \quad \forall x, y, z \in N .
$$

Putting $x r$ instead of $x$ in (27), we get

$$
F(y, z) g(x) d(r)=0, \quad \forall x, y, z, r \in N,
$$

which can be rewritten as

$$
F(y, z) N d(r)=\{0\}, \quad \forall y, z, r \in N \text {. }
$$

Since $N$ is a 3 -prime near ring and $d \neq 0$, we get

$$
F(y, z)=0, \quad \forall y, z \in N
$$

Replacing $y$ and $z$ by $r y$ and $r z$, respectively, in (30), we obtain

$$
\begin{aligned}
0 & =F(r(y, z)) \\
& =r F(y, z)+d(r) g(y, z) \\
& =d(r) g(y, z), \quad \forall y, z, r \in N .
\end{aligned}
$$

Taking $r t$ instead of $r$ in the last equation and using Lemma 5(ii), we get

$$
d(r) \operatorname{tg}(y, z)=0, \quad \forall y, z, r, t \in N .
$$

Thus,

$$
d(r) N g(y, z)=\{0\}, \quad \forall y, z, r \in N \text {. }
$$

Again using the fact that $N$ is 3-prime and $d \neq 0$, we find that $g(y)+g(z)=g(z)+g(y)$ for all $y, z \in N$. Since $g$ is onto, $(N,+)$ is abelian.

Theorem 11. Let $N$ be a 2-torsion free 3-prime near ring admitting a nonzero generalized semiderivation $F$ associated with a nonzero semiderivation $d$ and onto map $g$ associated with $d$ such that $g(x y)=g(x) g(y)$ for all $x, y \in N$. If $[F(N), F(N)]=0$, then $N$ is a commutative ring.

Proof. By the hypothesis

$$
F(x) F(y)=F(y) F(x), \quad \forall x, y \in N .
$$

Replace $y$ by $F(z) y$ in the above relation, and we get

$$
F(x) F(F(z) y)=F(F(z) y) F(x), \quad \forall x, y, z \in N .
$$

This implies that

$$
\begin{aligned}
F(x)(d(F(z)) g(y)+F(z) F(y)) & \\
=(d(F(z)) g(y)+F(z) F(y)) F(x), & \\
& \forall x, y, z \in N .
\end{aligned}
$$

Using Lemma 5(i), we find that

$$
\begin{aligned}
F(x) d(F(z)) g(y)=d(F(z)) g(y) F(x), & \\
& \forall x, y, z \in N .
\end{aligned}
$$

Taking $y w$ instead of $y$ in (37) and using (37)

$$
\begin{aligned}
& d(F(z)) g(y) F(x) g(w) \\
& \quad=d(F(z)) g(y) g(w) F(x), \quad \forall x, y, z, w \in N .
\end{aligned}
$$

Since $g$ is onto, we get

$$
d(F(z)) y F(x) w=d(F(z)) y w F(x)
$$

$$
\forall x, y, z, w \in N \text {. }
$$

This implies that

$$
d(F(z)) N(F(x) w-w F(x))=\{0\}, \quad \forall z, w \in N .
$$

Since $N$ is a 3-prime near ring, we have

$$
\begin{aligned}
& d(F(N))=\{0\} \\
& \text { or } F(N) \subseteq Z .
\end{aligned}
$$

If $F(N)$ is contained in $Z$, then $N$ is a commutative ring by Theorem 8. On the other hand, we see that if $d(F(N))=0$, then

$$
d(F(x y))=d(d(x) g(y)+x F(y))=0,
$$

$$
\forall x, y \in N \text {. }
$$

Thus

$$
\begin{gathered}
d^{2}(x) g(y)+d(x) d(g(y))+d(x) F(y) \\
+x d(F(y))=0, \quad \forall x, y \in N .
\end{gathered}
$$

This implies that

$$
\begin{aligned}
& d^{2}(x) g(y)+d(x) d(g(y))+d(x) F(y)=0, \\
& \forall x, y \in N .
\end{aligned}
$$


Replacing $y$ by $y z$ and using the fact that $g$ is onto, we get

$$
\begin{aligned}
& d^{2}(x) y z+d(x) d(y z)+d(x) F(y z)=0, \\
& \forall x, y, z \in N . \\
& d^{2}(x) y z+d(x)(y d(z)+d(y) g(z)) \\
& +d(x)(F(y) z+g(y) d(z))=0, \quad \forall x, y, z \in N, \\
& d^{2}(x) y z+d(x) y d(z)+d(x) d(y) g(z) \\
& +d(x) F(y) z+d(x) g(y) d(z)=0,
\end{aligned}
$$$$
\forall x, y, z \in N \text {, }
$$

$$
d^{2}(x) y z+d(x) y d(z)+d(x) d(y) z
$$$$
+d(x) F(y) z+d(x) y d(z)=0, \quad \forall x, y, z \in N,
$$$$
\left\{d^{2}(x) y+d(x) d(y)+d(x) F(y)\right\} z
$$$$
+2 d(x) y d(z)=0, \quad \forall x, y, z \in N \text {. }
$$

Since $N$ is 2-torsion free, using (44) we get

$$
\begin{aligned}
d(x) y d(z) & =0, \quad \forall x, y, z \in N, \\
d(N) N d(N) & =\{0\} .
\end{aligned}
$$

Thus we obtain that $d=0$, a contradiction which completes the proof.

Corollary 12 ([6, Theorem 4.1]). Let $N$ be a 2-torsion free prime near ring. If $N$ admits a generalized derivation $F$ associated with a nonzero derivation $d$ such that $[F(x), F(y)]=0$ for all $x, y \in N$, then $N$ is a commutative ring.

Theorem 13. Let $N$ be a 2-torsion free 3-prime near ring. If $F$ is a generalized semiderivation of $N$ associated with a nonzero semiderivation $d$ and an automorphism $g$ associated with $d$, then the following assertions are equivalent:

(i) $F([x, y])=[F(x), y]$ for all $x, y \in N$.

(ii) $F([x, y])=-[F(x), y]$ for all $x, y \in N$.

(iii) $N$ is a commutative ring.

Proof. It is obvious that (iii) implies both (i) and (ii).

Now we prove that (i) $\Rightarrow$ (iii). By hypothesis

$$
F([x, y])=[F(x), y], \quad \forall x, y \in N .
$$

Taking $x y$ instead of $y$ in (47) and noting that $[x, x y]=$ $x[x, y]$, we get

$$
\begin{array}{r}
x F([x, y])+d(x) g([x, y])=F(x) x y-x y F(x), \\
\forall x, y \in N .
\end{array}
$$

Using (47) and noting that $F(x) x=x F(x)$ by (47), then the last equation yields

$$
d(x) g([x, y])=0, \quad \forall x, y \in N .
$$

Since $g$ is an automorphism, we get

$$
d(x) g(x) g(y)=d(x) g(y) g(x), \quad \forall x, y \in N \text {. }
$$

Replacing $y$ by $y t$ in (50) and using (50), we arrive at

$$
d(x) y[x, t]=0, \quad \forall x, y, t \in N .
$$

This implies that

$$
d(x) N[x, t]=\{0\}, \quad \forall x, t \in N \text {. }
$$

3-primeness of $N$ yields that either $N \subseteq Z$ or $d(N)=\{0\}$. In both the cases $N$ is a commutative ring by Lemmas 3 and 4, respectively.

Using the similar techniques as above we can show that (ii) $\Rightarrow$ (iii).

Corollary 14 (see [7, Theorem 2.6]). Let $N$ be a 3-prime near ring. If $N$ admits a generalized derivation $F$ associated with a nonzero derivation $d$ such that $F([x, y])=[F(x), y]$ for all $x, y \in N$, then $N$ is a commutative ring.

Theorem 15. Let $N$ be a 2-torsion free 3-prime near ring. If $F$ is a generalized semiderivation of $N$ with associated semiderivation $d$ and an automorphism $g$ associated with $d$, then the following assertions are equivalent:

(i) $F([x, y])=[x, F(y)]$ for all $x, y \in N$.

(ii) $F([x, y])=-[x, F(y)]$ for all $x, y \in N$.

(iii) $N$ is a commutative ring.

Proof. Obviously, (iii) implies both (i) and (ii).

Now we prove that (i) $\Rightarrow$ (iii). By hypothesis

$$
F([x, y])=[x, F(y)], \quad \forall x, y \in N
$$

Replacing $x$ by $y x$ in (53), we arrive at

$$
\begin{array}{r}
y F([x, y])+d(y) g([x, y])=y x F(y)-F(y) y x, \\
\forall x, y \in N .
\end{array}
$$

Using (53) and noting that $y F(y)=F(y) y$ by (53), we find that

$$
d(y) g([x, y])=0, \quad \forall x, y \in N \text {. }
$$

Arguing in the similar manner as in Theorem 13, we get the result.

Similarly we can prove that (ii) $\Rightarrow$ (iii).

Corollary 16 (see [7, Theorem 2.7]). Let $N$ be 3-prime near ring. If $N$ admits a generalized derivation $F$ associated with a nonzero derivation $d$ such that $F([x, y])=[x, F(y)]$ for all $x, y \in N$, then $N$ is a commutative ring.

The following example shows that the conditions on the hypothesis of the above theorems are not superfluous. 
Example 17. Let $S$ be a 2-torsion free left near ring and let

$$
N=\left\{\left(\begin{array}{lll}
0 & 0 & x \\
0 & 0 & y \\
0 & 0 & 0
\end{array}\right) \mid x, y \in S\right\}
$$

Define $F, d, g: N \rightarrow N$ by

$$
\begin{aligned}
F\left(\begin{array}{lll}
0 & 0 & x \\
0 & 0 & y \\
0 & 0 & 0
\end{array}\right) & =\left(\begin{array}{lll}
0 & 0 & 0 \\
0 & 0 & y \\
0 & 0 & 0
\end{array}\right) ; \\
d\left(\begin{array}{lll}
0 & 0 & x \\
0 & 0 & y \\
0 & 0 & 0
\end{array}\right) & =\left(\begin{array}{lll}
0 & 0 & x \\
0 & 0 & 0 \\
0 & 0 & 0
\end{array}\right), \\
g\left(\begin{array}{lll}
0 & 0 & x \\
0 & 0 & y \\
0 & 0 & 0
\end{array}\right) & =\left(\begin{array}{lll}
0 & 0 & y \\
0 & 0 & x \\
0 & 0 & 0
\end{array}\right) .
\end{aligned}
$$

It can be checked that $N$ is a left near ring and $F$ is a generalized semiderivation of $N$ associated with a semiderivation $d$ and onto map $g$ associated with $d$ satisfying

(i) $[d(A), d(B)]=0$,

(ii) $F(N) \subseteq Z$,

(iii) $F([A, B])= \pm[F(A), B]$,

(iv) $F([A, B])= \pm[A, F(B)]$,

(v) $[F(A), F(B)]=0$

for all $A, B \in N$. However, $N$ is not a commutative ring.

\section{Generalized Semiderivations Acting as a Homomorphism or as an Antihomomorphism}

In [8], Bell and Kappe proved that if $R$ is a semiprime ring and $d$ is a derivation on $R$ which is either an endomorphism or an antiendomorphism on $R$, then $d=0$. Of course, derivations which are not endomorphisms or antiendomorphisms on $R$ may behave as such on certain subsets of $R$; for example, any derivation $d$ behaves as the zero endomorphism on the subring $C$ consisting of all constants (i.e., the elements $x$ for which $d(x)=0$ ). In fact in a semiprime $\operatorname{ring} R, d$ may behave as an endomorphism on a proper ideal of $R$. However as noted in [8], the behaviour of $d$ is somewhat restricted in the case of a prime ring. Recently the authors in [9] considered $(\theta, \phi)$-derivation $d$ acting as a homomorphism or an antihomomorphism on a nonzero Lie ideal of a prime ring and concluded that $d=0$. In this section we establish similar results in the setting of a 3-prime near ring admitting a generalized semiderivation.

Theorem 18. Let $N$ be a 3-prime near ring. Suppose that $F$ is a generalized semiderivation of $N$ associated with a semiderivation $d$ and onto map $g$ associated with $d$ such that $g(x y)=g(x) g(y)$ for all $x, y \in N$. If $F$ acts as $a$ homomorphism on $N$, then either $F$ is identity map or $F=0$.

Proof. By the hypothesis

$$
F(x y)=d(x) g(y)+x F(y)=F(x) F(y),
$$

$\forall x, y \in N$.

Replacing $y$ by $y z$ in the above relation, we get

$$
\begin{array}{cc}
F(x y z)=d(x) g(y z)+x F(y z), & \\
& \forall x, y, z \in N . \\
F(x y) F(z)=d(x) g(y z)+x F(y z), & \\
& \forall x, y, z \in N .
\end{array}
$$

This implies that

$$
\begin{aligned}
& (d(x) g(y)+x F(y)) F(z) \\
& \quad=d(x) g(y z)+x(d(y) g(z)+y F(z)),
\end{aligned}
$$

$$
\forall x, y, z \in N \text {. }
$$

Using Lemma 5(ii), we obtain

$$
\begin{array}{cc}
d(x) & g(y) F(z)+x F(y) F(z) \\
=d(x) g(y z)+x d(y) g(z)+x y F(z), & \forall x, y, z \in N . \\
d(x) g(y) F(z)+x F(y z) & \forall x, y, z \in N . \\
=d(x) g(y z)+x d(y) g(z)+x y F(z), & \\
d(x) g(y) F(z)+x d(y) g(z)+x y F(z) & \forall x, y, z \in N .
\end{array}
$$

This implies that

$$
\begin{aligned}
d(x) g(y) F(z)=d(x) g(y) & g(z), \\
& \forall x, y, z \in N,
\end{aligned}
$$

that is $d(x) y(F(z)-z)=0, \quad \forall x, y, z \in N$.

Thus

$$
d(x) N(F(z)-z)=\{0\}, \quad \forall x, y, z \in N .
$$

Therefore, $d(N)=\{0\}$ or $F(z)=z$ for all $z \in N$.

In the later case $F$ is an identity map. On the other hand suppose that $d(N)=\{0\}$. Then $F(x y)=F(x) y=F(x) F(y)$; that is, $F(x)(y-F(y))=0$ for all $x, y \in N$. Replacing $y$ by $z y$, $z \in N$, and noting that $F(z y)=z F(y)$, we have $F(x) N(y-$ $F(y))=\{0\}$ for all $x, y \in N$. Therefore, $F(N)=\{0\}$ or $F$ is an identity map. 
Theorem 19. Let $N$ be a 2-torsion free 3-prime near ring. Suppose that $F$ is a generalized semiderivation of $N$ associated with a semiderivation $d$ and onto map $g$ such that $g(x y)=$ $g(x) g(y)$ for all $x, y \in N$. If $F$ acts as antihomomorphism on $N$, then $F=0$ or $F$ is the identity map on $N$ and $N$ is a commutative ring.

Proof. By the hypothesis

$$
\begin{aligned}
F(x y)=d(x) g(y)+x F(y)=F(y) F(x), & \\
& \forall x, y \in N .
\end{aligned}
$$

Thus

$$
F(y) F(x)=d(x) g(y)+x F(y), \quad \forall x, y \in N \text {. }
$$

Replacing $y$ by $x y$ in the above relation, we obtain

$$
\begin{aligned}
& F(x y) F(x)=d(x) g(x y)+x F(x y), \quad \forall x, y \in N, \\
& \text { that is }(d(x) g(y)+x F(y)) F(x) \\
& \quad=d(x) g(x y)+x F(y), \quad \forall x, y \in N .
\end{aligned}
$$

By Lemma 5(ii), we have

$$
\begin{aligned}
& d(x) g(y) F(x)+x F(y) F(x) \\
& \quad=d(x) g(x y)+x F(y), \quad \forall x, y \in N .
\end{aligned}
$$

This implies that

$$
d(x) g(y) F(x)=d(x) g(x) g(y), \quad \forall x, y \in N .
$$

Replacing $y$ by $y r$ in the above relation, we get

$$
\begin{array}{r}
d(x) g(y) g(r) F(x)=d(x) g(x) g(y) g(r), \\
\forall x, y, r \in N .
\end{array}
$$

Using (68) in the above relation, we get

$$
\begin{aligned}
d(x) g(y) g(r) F(x)=d(x) g(y) F(x) & g(r), \\
& \forall x, y, r \in N .
\end{aligned}
$$

Since $g$ is onto, we have

$$
d(x) N[F(x), r]=\{0\}, \quad \forall x, r \in N
$$

Therefore, either $d(N)=\{0\}$ or $F(N) \subseteq Z$. Hence in either case $F$ acts as a homomorphism by Lemma 4 and Theorem 8 which completes the proof.

\section{Conflict of Interests}

The authors declare that there is no conflict of interests regarding the publication of this paper.

\section{References}

[1] J. Bergen, "Derivations in prime rings," Canadian Mathematical Bulletin, vol. 26, no. 3, pp. 267-270, 1983.

[2] H. E. Bell and G. Mason, "On derivations in near-rings," NorthHolland Mathematics Studies, vol. 137, pp. 31-35, 1987.

[3] A. Boua and L. Oukhtite, "Semiderivations satisfying certain algebraic identities on prime near-rings," Asian-European Journal of Mathematics, vol. 6, no. 3, Article ID 1350043, 8 pages, 2013.

[4] I. N. Herstein, "A note on derivations," Canadian Mathematical Bulletin, vol. 21, pp. 369-370, 1978.

[5] H. E. Bell, "On derivations in near-rings, II," in Nearrings, Nearfields and K-Loops, vol. 426 of Mathematics and Its Applications, pp. 191-197, Springer, Dordrecht, The Netherlands, 1997.

[6] H. E. Bell, "On prime near-rings with generalized derivation," International Journal of Mathematics and Mathematical Sciences, vol. 2008, Article ID 490316, 5 pages, 2008.

[7] A. Boua and L. Oukhtite, "Some conditions under which nearrings are rings," Southeast Asian Bulletin of Mathematics, vol. 37, no. 3, pp. 325-331, 2013.

[8] H. E. Bell and L.-C. Kappe, "Rings in which derivations satisfy certain algebraic conditions," Acta Mathematica Hungarica, vol. 53, no. 3-4, pp. 339-345, 1989.

[9] A. Asma, N. Rehman, and A. Shakir, "On Lie ideals with derivations as homomorphisms and anti-homomorphisms," Acta Mathematica Hungarica, vol. 101, no. 1-2, pp. 79-82, 2003. 


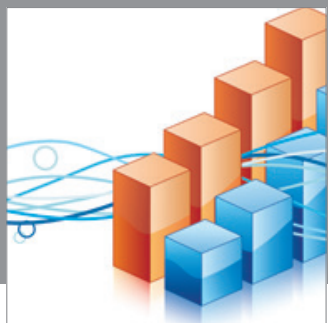

Advances in

Operations Research

mansans

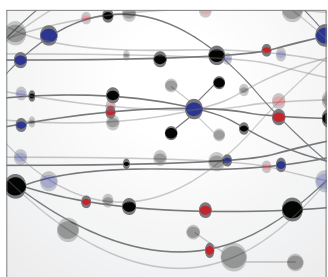

The Scientific World Journal
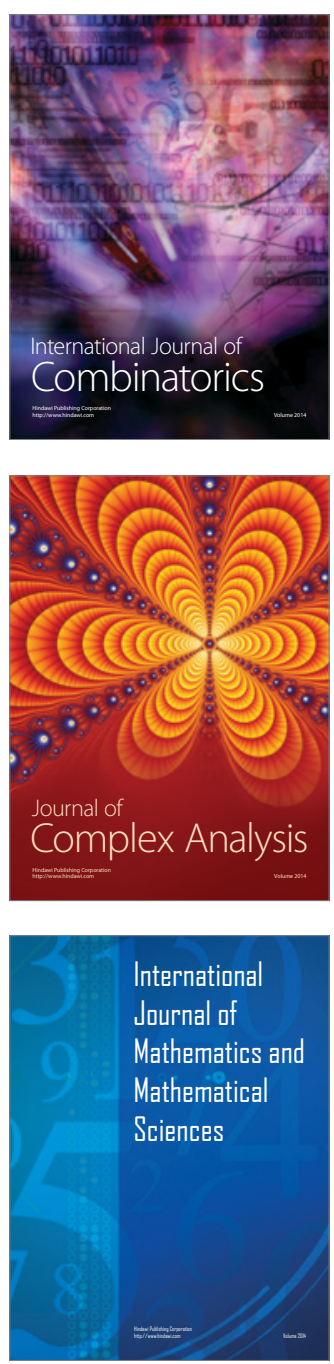
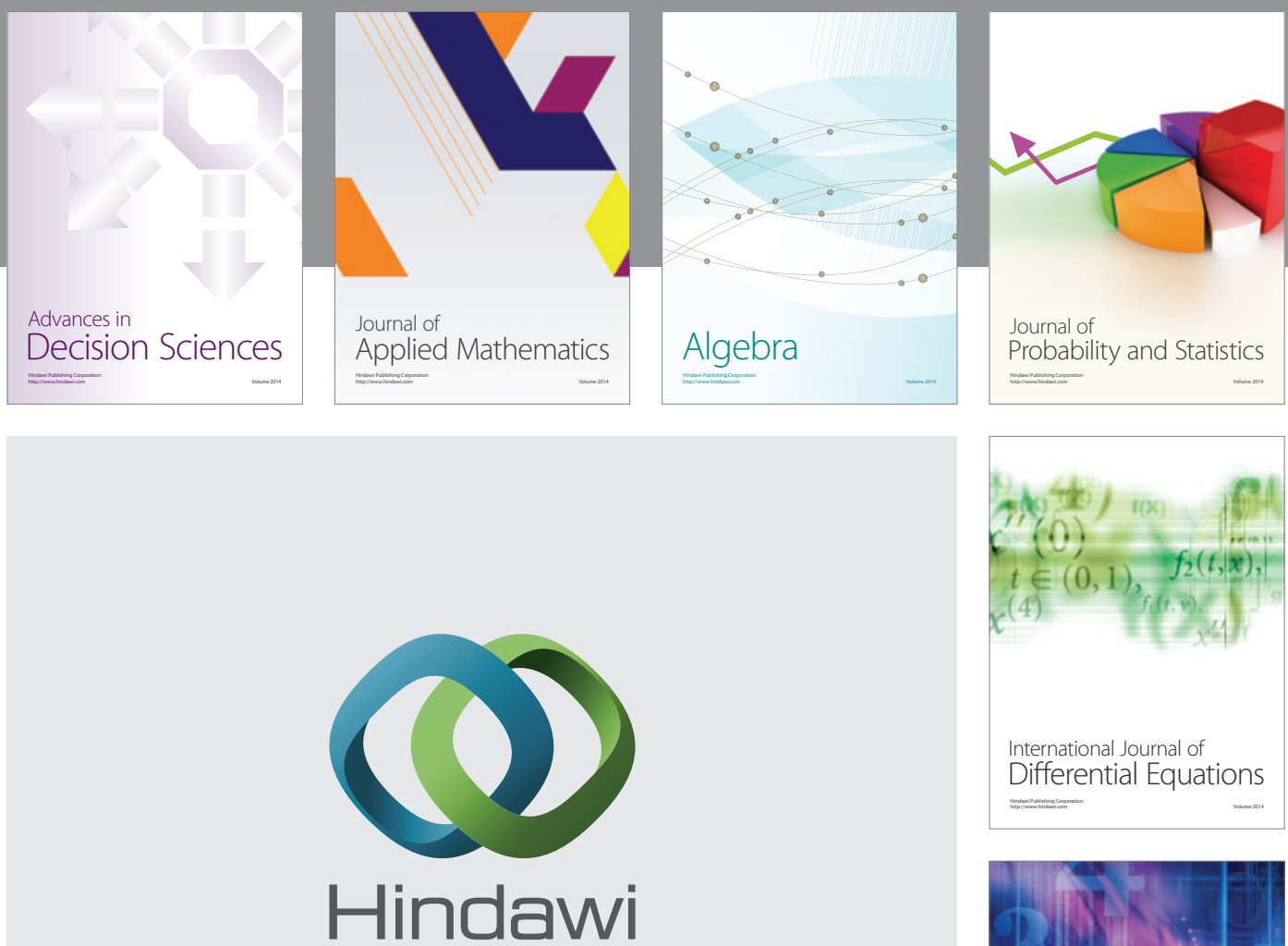

Submit your manuscripts at http://www.hindawi.com
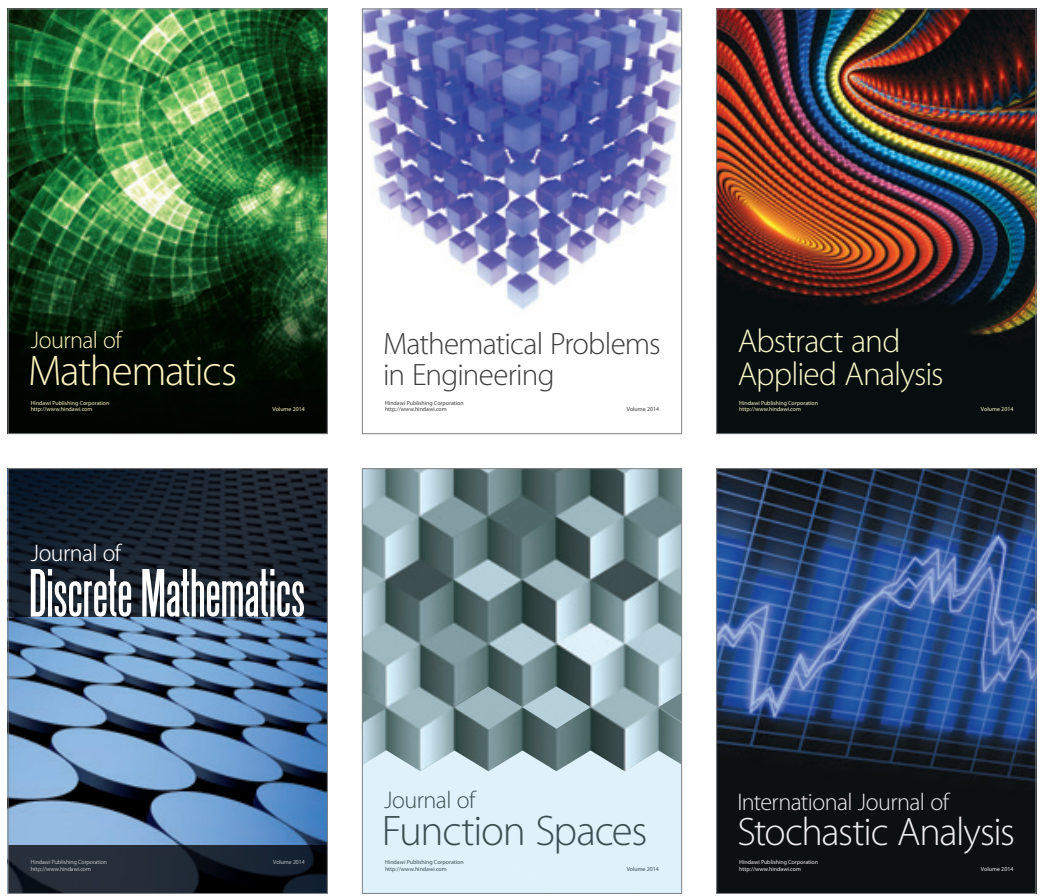

Journal of

Function Spaces

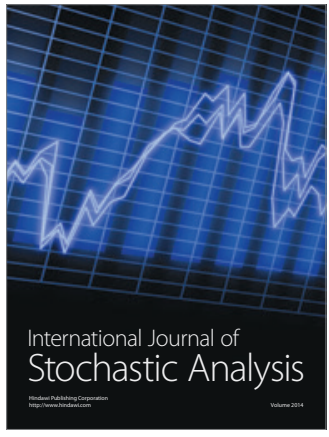

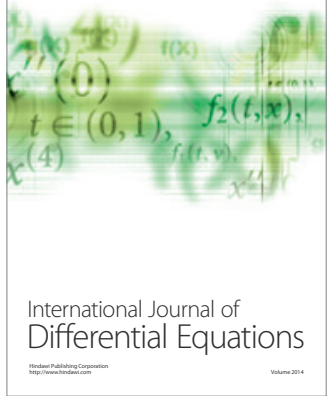
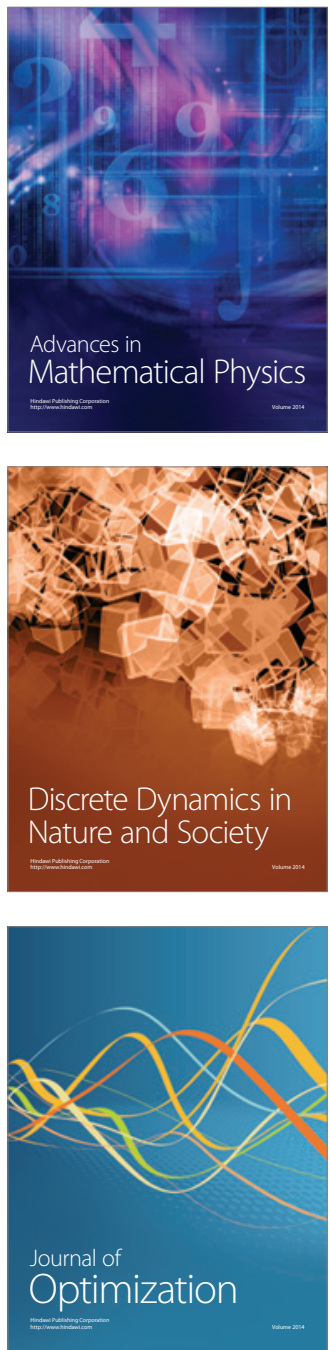